\title{
DOCENTES Y TIC: UN ENCUENTRO NECESARIO
}

\author{
Ignacio Berzosa Ramos \\ María José Arroyo González \\ Universidad de Valladolid
}

\begin{abstract}
RESUMEN: Las TIC (Tecnologías de Información y la Comunicación) constituyen un elemento determinante en la sociedad actual y que van, poco a poco, incorporándose en la escuela. En ese proceso de integración los docentes toman un papel preponderante que es necesario investigar. En el presente artículo se revisa la manera en que la integración de las TIC en un centro educativo concreto afecta a las actitudes de los docentes hacia las mismas, al tiempo que se observa la evolución que los docentes experimentan en los usos que hacen de las TIC.
\end{abstract}

PALABRAS CLAVE: Educación, TIC, profesorado, actitudes, usos.

\section{TEACHERS AND ICT: A NECESSARY MEETING}

ABSTRACT: ICT (Information and Communication) are a key element in today's society and they will, little by little, sitting in the school. In this integration process teachers take a leading role research is needed. In this article the way the integration of ICT in a particular school affects the attitudes of teachers towards the same is reviewed, while the evolution that teachers experience in the uses made of ICT is observed and how they become an innovative tool that helps in the search for new educational practices.

KEYWORDS: Education, ICT, teachers, attitudes, uses.

Recibido: 30/04/2015

Aceptado: 14/07/2015

Correspondencia: María José Arroyo González, Dpto. de Pedagogía, Facultad de Educación, Campus María Zambrano, Universidad de Valladolid, Plaza de la Universidad 1, 40005 Segovia. Email: mjarroyo@edu.ucm.es. 


\section{INTRODUCCIÓN}

Las Tecnologías de información y la Comunicación (TIC) están configurando una nueva sociedad. Ante esta nueva realidad aparece el importante reto de poder definir una forma de vivir de forma crítica este nuevo modelo social que está desarrollándose. Si concebimos que la labor última de la educación debe ser formar ciudadanos críticos, no sólo trabajadores cualificados, el reto que en estos momentos se nos plantea, exige de la escuela, (entendida como cualquier institución de educación formal) un verdadero cambio profundo que la sitúe a la vanguardia de esta nueva sociedad y que la permita cumplir la labor de transformación que debe tener.

En este contexto, las TIC pueden ser una excusa, un instrumento, para el cambio educativo necesario. Por sí solas, las TIC no aportan innovación si no van unidas a nuevas formas de organizar y plantear los aprendizajes, pero sí que pueden ser herramientas imprescindibles a la hora de plantear una innovación educativa auténtica y perdurable en el tiempo.

Aunque son múltiples los factores que influyen en el proceso de integración de las TIC dentro de los sistemas educativos, a nadie se le escapa la importancia que tienen los docentes en esta realidad, por eso es importante detenernos a estudiar su papel.

Si creemos, como afirma Díez (1998), que el profesorado es pieza clave para conseguir la calidad de la educación (y nosotros lo creemos), es lógico pensar que cualquier aspecto que vaya a incidir de manera directa en las aulas debe pasar por reconocer la importancia del profesorado para que esa realidad se plasme.

En esa escuela desafiada por los acontecimientos e interpelada por esta nueva sociedad está el docente. En muchos momentos desorientado y fuera de lugar. Profesorado que debe afrontar al enorme problema de que "la sociedad ha cambiado muy rápido y tiene esquemas, métodos y materiales que no puede aplicar" (Area, Gros y Marzal, 2008: 170).

La investigación aquí presentada forma parte de una experiencia más amplia sobre lo que es una aproximación concreta y aplicada del proceso de integración curricular de las TIC en un centro educativo. Esta experiencia se basa en las premisas de la Investigación-Acción y parte de una concepción muy concreta de lo que significa la investigación educativa (Elliot, 1990), ya que tal y como afirman Carr y Kemmis (1998):

La piedra de toque de la investigación educativa no será su refinamiento teórico ni su capacidad para satisfacer criterios derivados de las ciencias sociales, sino, por encima de todo, su capacidad para resolver problemas educacionales y mejora la práctica de la educación (p. 122).

El objetivo de esta investigación que aquí se presenta es mostrar la evolución que muestran los docentes de un centro concreto respecto a las actitudes hacia las TIC y el uso que hacen de las mismas en su labor docente. La información obtenida debe servir para diseñar procesos más ajustados de intervención en el claustro de este centro (como por ejemplo en formación), para poder alcanzar de manera más adecuada el fin último de la investigación que no es otro que optimizar el proceso de integración de las TIC. 


\section{LOS DOCENTES EN LA INTEGRACIÓN ESCOLAR DE LAS TIC}

Hoy en día nadie duda de las posibilidades educativas de las TIC, y esto nos hace plantearnos como docentes, las maneras de ser y de hacer las cosas. En este sentido, creemos que se está produciendo un cambio importante en la actitud ante las TIC y en la manera de aplicarlas didácticamente y de aprovechar su valor de comunicación.

Y sin embargo, a pesar de los esfuerzos para que las TIC se integren en las escuelas mediante políticas y planes, las expectativas generadas parecen no cumplirse (TiradoMorueta y Aguaded-Gómez, 2014). Como recogen los citados autores, numerosos estudios se han puesto en marcha para identificar las razones de esta situación, Ilegándose a plantear modelos que explican la integración de las TIC en las escuelas desde la combinación de diversas variables. Podemos hablar de la existencia de factores de primer orden (extrínsecos al aula) y de factores de segundo orden (intrínsecos) (Colás y Casanova, 2010). Estos factores de segundo orden hacen referencia directa al profesor y entre los más destacados estarían las actitudes y creencias de los docentes hacia las TIC (Tirado-Morueta y Aguaded-Gómez, 2014).

Desde ya hace algunos años, se ha indagado profusamente sobre cómo afectan los cambios que está experimentando la escuela y en concreto en relación con la integración de las TIC, al profesorado y también sobre cómo es su nivel de aceptación de los mismos. Como nos resumen diversos autores (Tejedor, García-Valcarcel y Prada, 2009; Tirado-Morueta y Aguaded-Gómez, 2014) se han desarrollado múltiples investigaciones en diversas líneas y centradas en distintas problemáticas.

En particular, las referidas a los aspectos actitudinales se centran en temas como los siguientes: cómo las actitudes hacia los ordenadores condicionan el tipo y forma de interacción entre usuarios y ordenadores, cómo estas interacciones modifican las actitudes iniciales hacia los mismos, qué variables determinan o condicionan las actitudes de los usuarios hacia los ordenadores y contraste entre las actitudes que profesores y alumnos tienen hacia los ordenadores (Inan y Lowther, 2010; Ertmer, 2005; Hew y Brush, 2007).

En los estudios realizados hasta la mitad de la década pasada podemos observar que las actitudes expresadas por los docentes se ubicarían entre dos extremos: la actitud de quienes sienten una amenaza a su rutina y en el otro la de quienes tienen una actitud positiva pues ven en las TIC un agente motivador en el proceso de aprendizaje de los alumnos.

Frente a esta dicotomía observada en esos primeros años de irrupción de las TIC, es posible observar ahora un cambio de actitud. Creemos que se han suavizado las posiciones extremas, especialmente las de los docentes más refractarios al uso de las TIC, aunque mucho nos tememos, y esto es una reflexión personal, que no por convencimiento, sino por pura adaptación. Además en la mayoría de los casos, las prácticas docentes no han cambiado, lo único que se han modificado son las herramientas (Fidalgo, 2011), en definitiva se han mantenido las viejas pedagogías con nuevas herramientas (usar un cañón proyector en vez de la pizarra y la tiza, no es una gran evolución en la forma en que la clase se desarrolla y lo que sucede en ella). Porque, ciertamente, lo que el docente piensa sobre el potencial didáctico de las TIC condiciona su uso en la práctica docente (Tejedor, García-Valcárcel y Prada, 2009). 
Pero cuando se consideran las actitudes hacia las TIC de los docentes deben tenerse en cuenta otras variables que interaccionan de manera diferente; unas se refieren a la propia naturaleza de la tecnología, otras a los usuarios, las referidas al medio escolar y el resto de elementos curriculares y por último, a las que involucran al contexto de aprendizaje. Por ejemplo, Tejedor, García-Valcarcel y Prada (2009) indican que las actitudes del profesorado ante la introducción de las TIC en la educación tienen mucho que ver con las creencias sobre los beneficios educativos de estos medios y, sobre todo, con la propia autoestima. También son generadoras de actitudes negativas de resistencia al cambio, el hecho de que no haya evidencias sobre la efectividad real.

Otros aspectos problemáticos que se siguen contemplando sobre el uso de las TIC por parte profesorado, y que destacan Calderón y Piñeiro (2007) son de resistencia al cambio, de deficiencias formativas en uso de tecnología, y de autoestima y grado de frustración del docente.

Como resumen final podríamos hablar de que existen tres figuras estereotipadas del docente en relación con las TIC (Tirado-Morueta, y Aguaded-Gómez, 2014):

- Dentro del ejercicio de la profesión, podemos encontramos docentes que muestran desarrollada la competencia digital, que utilizan las TIC en el aula de manera cotidiana y se atreve con la creación de recursos didácticos con las TIC, esforzándose por sacar el máximo rendimiento a éstas en su labor docente.

- Otros docentes, aun habiendo adquirido la competencia digital, no buscan nuevos enfoques de uso de las TIC en el aula y se acomodan en un uso meramente instrumental sin incidir en la calidad de la enseñanza dentro de la era digital.

- Por último, encontramos docentes que no tienen en absoluto desarrollada la competencia digital. Esta circunstancia puede venir dada por motivos diversos. Por ejemplo la desmotivación que le provoca la sensación de no ser capaz de adaptarse a un mundo complejo y en constante evolución como es la realidad de las TIC, o a la sensación de encontrarse en inferioridad en el uso de herramientas que los propios alumnos, nativos digitales, sabrán manejar siempre mejor que él mismo. La inseguridad que le provocan todos estos factores, le hacen impermeable a la adquisición y puesta en práctica de estas competencias. En otras ocasiones, el rechazo viene derivado del desconocimiento de las posibilidades y posibles usos que pueden tener las TIC. Y por último, nos podemos encontrar con auténticos casos de tecnofobia (Gros, 2000).

En cualquier caso, la motivación por aprender es indiscutiblemente la base para poder llevar a cabo cualquier tipo de innovación o proyecto de integración curricular de las TIC en los contestos educativos.

Estas actitudes negativas, unidas a bajos niveles de competencia digital nos dibujan un perfil de profesores que parten con un hándicap en el desempeño de su profesión al no tener adquiridos conocimientos ni destrezas para acceder a la información y generarla en distintos lenguajes (Area, 2012). 
Como queda claro, la formación es uno de los elementos determinantes de la actitud que poseen los docentes hacia las TIC y una de las líneas de teorización más fructíferas en el campo de la formación del profesorado en los últimos años ha sido el intento por definir las competencias que un docente debe tener para desempeñar de manera adecuada su labor en este siglo XXI.

En este contexto, no podemos dejar de mencionar uno de los proyectos clave del Plan de Cultura Digital en la Escuela del Ministerio de Educación, Cultura y Deporte, como es el "Marco Común de Competencia Digital Docente" (INTEF, 2014). Aunque aún en fase de discusión, en febrero de 2014 fue presentado durante una sesión de trabajo celebrada en Valladolid un primer borrador del citado marco.

Los objetivos marcados para el proyecto son:

- Posibilitar que los profesores conozcan, ayuden a desarrollar y evalúen la competencia digital de los alumnos.

- Facilitar una referencia común con descriptores de la competencia digital para profesores y formadores.

- Ayudar a que el docente tenga la competencia digital necesaria para usar recursos digitales en sus tareas docentes.

- Influir para que se produzca un cambio metodológico tanto en el uso de los medios tecnológicos como en los métodos educativos en general (INTEF, 2014).

La definición y características de la competencia digital docente propuesta en dicho borrador se basa en la competencia digital del proyecto DIGCOMP (Digital Competence: Identification and European-wide validation of its key components for all levels of learners) desarrollado por el IPTS (Instituto de Prospectiva Tecnológica).

En el borrador se marcan una serie "áreas de competencia digital" que pueden ser resumidas de la siguiente manera:

- Información: identificar, localizar, recuperar, almacenar, organizar y analizar la información digital, evaluando su finalidad y relevancia.

- Comunicación: comunicar en entornos digitales, compartir recursos a través de herramientas en línea, conectar y colaborar con otros a través de herramientas digitales, interactuar y participar en comunidades y redes; conciencia intercultural.

- Creación de contenido: Crear y editar contenidos nuevos (textos, imágenes, videos...), integrar y reelaborar conocimientos y contenidos previos, realizar producciones artísticas, contenidos multimedia y programación informática, saber aplicar los derechos de propiedad intelectual y las licencias de uso.

- Seguridad: protección personal, protección de datos, protección de la identidad digital, uso de seguridad, uso seguro y sostenible.

- Resolución de problemas: identificar necesidades y recursos digitales, tomar decisiones a la hora de elegir la herramienta digital apropiada, acorde a la finalidad o necesidad, resolver problemas conceptuales a través de medios digitales, resolver problemas técnicos, uso creativo de la tecnología, actualizar la competencia propia y la de otros (INTEF, 2014). 
Este proyecto puede ser una buena oportunidad para marcar unas directrices comunes y consensuadas acerca de las competencias relacionadas con TIC que los docentes deben poseer, para de esta manera reorientar los planes de estudio de los futuros educadores y también perfilar las líneas maestras de la formación permanente en TIC de los docentes en activo.

\section{MATERIAles y MÉTOdos}

Como indicábamos anteriormente, esta investigación se encuadra dentro de un proceso de Investigación-Acción sobre la integración de las TIC en un centro docente concreto. Este centro educativo, situado en la ciudad de Segovia, abarca desde Infantil hasta Bachillerato y posee en la actualidad un claustro de 103 profesores. Son estos docentes en su conjunto sobre los que planteamos el estudio, por eso, para poder llegar a todos los profesores, utilizamos el cuestionario como herramienta para recabar la información.

Para nuestro trabajo se utilizaron dos cuestionarios diferentes. El primero se pasó en mayo de 2010, mientras que el segundo se contestó en abril de 2014. Este último se basó en el primero, aunque fue rectificado en diversos aspectos, pero con varios ítems repetidos.

A partir de la información preliminar obtenida con el análisis documental, se elaboró un primer cuestionario, que fue revisado y depurado sucesivamente, buscando una mayor claridad en las cuestiones y también una mejor confiabilidad del test.

Una característica importante de los cuestionarios es el tipo de respuesta que obtenemos; en nuestro caso se formularon preguntas abiertas, cerradas y mixtas, aunque las más utilizadas fueron las preguntas cerradas de opción múltiple y las que pedían el posicionamiento de la respuesta en una escala. Este último tipo de ítem es especialmente importante a la hora de plantearnos una aproximación a las actitudes de los docentes hacia las TIC, y en esos casos se utilizaron escalas tipo Liker.

El primer cuestionario desarrollado presentaba 21 preguntas, 19 de ellas cerradas con varias opciones y 2 abiertas. Se ha estructurado en 7 dimensiones, tal como queda reflejado en la Tabla 1. Para su elaboración nos basamos en cuestionarios anteriores de carácter similar.

Tabla 1. Dimensiones del primer cuestionario

\begin{tabular}{|c|l|c|}
\hline \multicolumn{1}{|c|}{ DIMENSIONES } & No ITEM $^{-1}$ \\
\hline 1. & Datos del profesor & $1,2,3,4,5$ \\
\hline 2. & Acceso y uso de TIC en el centro educativo & $6,7,8,9$ \\
\hline 3. & Acceso y uso de TIC en el hogar & 10,11 \\
\hline 4. & Formación del profesorado en TIC & $12,13,14$ \\
\hline 5. & Competencia percibida en el uso de las TIC & 15,16 \\
\hline 6. & Actitudes del profesorado hacia las TIC & $17,18,19,20,21$ \\
\hline 7. & Uso de la plataforma Educ@mos & \\
\hline
\end{tabular}


La consistencia interna es la vía escogida para cerciorarnos de la fiabilidad que presenta la herramienta diseñada y para obtener una estimación de la misma, nos decantamos por basarnos en el alfa de Cronbach como indicador. Para el cálculo de este índice recurrimos al uso del paquete estadístico SPSS, que nos permitió obtener unos valores finales comprendidos entre 0.7342 y 0.9451 (ver Tabla 2).

Tabla 2. Valores finales del índice Alfa de Cronbach para el Primer cuestionario

\begin{tabular}{|l|l|}
\hline \multicolumn{1}{|c|}{ DIMENSIONES } & \\
\hline Acceso y uso de TIC en el centro educativo & 0.7965 \\
\hline Acceso y uso de TIC en el hogar & 0.7342 \\
\hline Formación del profesorado en TIC & 0.8641 \\
\hline Competencia percibida en el uso de las TIC & 0.9012 \\
\hline Actitudes del profesorado hacia las TIC & 0.9451 \\
\hline Uso de la plataforma Educ@mos & 0.8794 \\
\hline
\end{tabular}

Como se ha indicado previamente, este segundo cuestionario era básicamente el mismo que el primero, aunque realizamos algunas modificaciones en el mismo que buscaban simplificar y acortar el cuestionario, potenciar aquellos ítems relacionados con las actitudes del profesorado hacia las TIC y eliminar ítems que aportaban poca información o esta era poco significativa: por ejemplo se eliminaron los ítems relacionados con la formación del profesorado, ya que era de suponer poca variación en los aspectos que trataban esos ítems.

Después de este proceso de revisión el cuestionario quedó con seis dimensiones, una menos que en el cuestionario anterior y con 12 ítems (ver Tabla 3).

Tabla 3. Dimensiones del segundo cuestionario

\begin{tabular}{|l|l|c|}
\hline & \multicolumn{1}{|c|}{ DIMENSIONES } & No ITEM $^{-1}$ \\
\hline 1. & Datos del profesor & $1,2,3,4,5,6$ \\
\hline 2. & Acceso y uso de TIC en el centro educativo & 7 \\
\hline 3. & Formación del profesorado en TIC & 8 \\
\hline 4. & Competencia percibida en el uso de las TIC & 9,10 \\
\hline $\mathbf{5 .}$ & Actitudes del profesorado hacia las TIC & 11,12 \\
\hline 6. & Uso de la plataforma Educ@mos & \\
\hline
\end{tabular}

Ambos cuestionarios, tanto el primero, como el segundo fueron ideados para que fueran contestados por todo el claustro del centro, por tanto no hubo ningún tipo de elección de muestra. Finalmente fueron recogidas las respuestas de 85 docentes para 
el primero de los cuestionarios y de 63, para el segundo. Dado el objetivo del estudio, nuestra población se reduce al conjunto de profesores del claustro, lo que puede repercutir en la confiabilidad estadística de las respuestas obtenidas.

\section{Resultados}

Una idea que nos parecía interesante desde un principio era la de poder realizar un pequeño estudio longitudinal, comparando los resultados obtenidos en ambos cuestionarios, siempre en función de poder mejorar el proceso de integración de las TIC en el centro. Dado que el número de respuestas era diferente en ambos cuestionarios, se procedió a transformar los datos para expresarlos en un porcentaje del total y que así fueran datos comparables.

En primer lugar presentamos la evolución en la frecuencia de uso de los medios usados por los profesores en su práctica docente con los alumnos. Los resultados obtenidos se pueden observar en la Tabla 4.

Tabla 4. Medios técnicos usados con los alumnos. Frecuencia de uso

\begin{tabular}{|c|c|c|c|c|c|c|c|c|}
\hline & \multicolumn{2}{|c|}{ Nunca } & \multicolumn{2}{|c|}{$\begin{array}{l}\text { Menos de una al } \\
\text { mes }\end{array}$} & \multicolumn{2}{|c|}{$\begin{array}{c}\text { Entre una a la } \\
\text { semana y una al } \\
\text { mes }\end{array}$} & \multicolumn{2}{|c|}{ Todas las semanas } \\
\hline & 2010 & 2014 & 2010 & 2014 & 2010 & 2014 & 2010 & 2014 \\
\hline Sala de informática & $75,56 \%$ & $37,04 \%$ & $15,56 \%$ & $44,44 \%$ & $2,22 \%$ & $11,11 \%$ & $6,67 \%$ & $7,41 \%$ \\
\hline DVD y Vídeo & $26,67 \%$ & $10,71 \%$ & $31,11 \%$ & $28,57 \%$ & $40,00 \%$ & $53,57 \%$ & $2,22 \%$ & $7,14 \%$ \\
\hline Ordenador del aula & $8,89 \%$ & $3,33 \%$ & $8,89 \%$ & $6,67 \%$ & $26,67 \%$ & $16,67 \%$ & $55,56 \%$ & $73,33 \%$ \\
\hline Pizarra digital & $79,55 \%$ & $59,26 \%$ & $11,36 \%$ & $29,63 \%$ & $9,09 \%$ & $3,70 \%$ & $0 \%$ & $7,41 \%$ \\
\hline Cañón proyector & $48,89 \%$ & $7,69 \%$ & $26,67 \%$ & $11,54 \%$ & $13,33 \%$ & $21.35 \%$ & $11.11 \%$ & $59.42 \%$ \\
\hline
\end{tabular}

Se puede apreciar un aumento en el uso general de todas las tecnologías, excepto el DVD, algo esperable. El ordenador de aula y el cañón proyector son los elementos que presentan un aumento mayor en su utilización en estos cuatro años. Destacamos el bajo uso de las salas de informática, que aunque si aumenta ligeramente el porcentaje de uso, sigue siendo muy esporádico.

En segundo lugar presentamos la evolución en los usos de las TIC por parte del profesorado (Tabla 5). Además se indica la frecuencia con que se realiza ese uso.

De lo recogido en el siguiente cuadro podemos apreciar un aumento muy significativo en los usos de las TIC por parte del profesorado. Algunos de ellos en concreto se han convertido en habituales para una gran mayoría de los docentes: buscar información mediante internet, utilizar procesadores de texto, gestionar el trabajo personal o su uso como herramienta de comunicación con alumnado y familias. Sin embargo, cabe señalar nuevamente que los usos que más han crecido y que son mayoritarios no son los que tienen que ver con el uso didáctico de las TIC por parte del profesorado en las aulas. Podemos pensar que esa es una asignatura pendiente para la integración de las TIC. 
Tabla 5. Medios técnicos usados con los alumnos. Frecuencia de uso

\begin{tabular}{|c|c|c|c|c|c|c|}
\hline & \multicolumn{2}{|c|}{$\begin{array}{l}\text { Menos de una al } \\
\text { mes }\end{array}$} & \multicolumn{2}{|c|}{$\begin{array}{c}\text { Entre una a la } \\
\text { semana y una al } \\
\text { mes }\end{array}$} & \multicolumn{2}{|c|}{ Todos los días } \\
\hline & 2010 & 2014 & 2010 & 2014 & 2010 & 2014 \\
\hline Usar procesador de texto & $11,63 \%$ & $3,57 \%$ & $16,28 \%$ & $17,86 \%$ & $72,09 \%$ & $78,57 \%$ \\
\hline $\begin{array}{l}\text { Navegar por Internet para buscar infor- } \\
\text { mación }\end{array}$ & $4,44 \%$ & $3,57 \%$ & $20,00 \%$ & $10,71 \%$ & $75,56 \%$ & $85,71 \%$ \\
\hline Gestionar el trabajo personal & $13,33 \%$ & $7,14 \%$ & $26,67 \%$ & $21,43 \%$ & $60 \%$ & $71,43 \%$ \\
\hline Usar el ordenador como apoyo a las clases & $18,18 \%$ & $7,14 \%$ & $52,27 \%$ & $46,43 \%$ & $29,55 \%$ & $46,43 \%$ \\
\hline Usar programas educativos & $25,58 \%$ & $28,57 \%$ & $60,47 \%$ & $50 \%$ & $13,95 \%$ & $21,43 \%$ \\
\hline Usar programas de diseño & $76,19 \%$ & $64.29 \%$ & $19,05 \%$ & $21,43 \%$ & $4,76 \%$ & $14,29 \%$ \\
\hline $\begin{array}{l}\text { Usar Internet para trabajos en grupo de } \\
\text { los alumnos }\end{array}$ & $59,09 \%$ & $42,86 \%$ & $31,82 \%$ & $35,71 \%$ & $9,09 \%$ & $21,43 \%$ \\
\hline Usar hojas de cálculo & $72,09 \%$ & $64,29 \%$ & $16,28 \%$ & $28,57 \%$ & $11,63 \%$ & $7,14 \%$ \\
\hline Descargar software educativo de Internet & $55,81 \%$ & $57,14 \%$ & $41,86 \%$ & $35,71 \%$ & $2,33 \%$ & $7,14 \%$ \\
\hline Hacer presentación y simulaciones & $72,09 \%$ & $35,71 \%$ & $13,95 \%$ & $39,29 \%$ & $13,95 \%$ & $25 \%$ \\
\hline Evaluar al alumnado & $29,55 \%$ & $28,57 \%$ & $40,91 \%$ & $32,14 \%$ & $29,55 \%$ & $39,29 \%$ \\
\hline Comunicarse con alumnos, padres... & $18,18 \%$ & $7,14 \%$ & $50 \%$ & $32,14 \%$ & $31,82 \%$ & $60,71 \%$ \\
\hline $\begin{array}{l}\text { Usar software multimedia relacionado } \\
\text { con contenidos curriculares }\end{array}$ & $51,16 \%$ & $32,14 \%$ & $44,19 \%$ & $39,29 \%$ & $4,65 \%$ & $28,57 \%$ \\
\hline $\begin{array}{l}\text { Usar software multimedia con conteni- } \\
\text { dos de referencia y consulta }\end{array}$ & $58,14 \%$ & $35,71 \%$ & $34,88 \%$ & $39,29 \%$ & $6,98 \%$ & $25 \%$ \\
\hline
\end{tabular}

La Tabla 6 recoge las respuestas obtenidas a la pregunta de si utilizas software educativo y contenidos digitales en tu tarea docente y como se puede apreciar hay un aumento muy significativo en su uso en estos dos años transcurridos.

Tabla 6. Uso de Software educativo y contenidos digitales

\begin{tabular}{|c|c|c|c|}
\hline \multicolumn{2}{|c|}{ SI } & \multicolumn{2}{c|}{ NO } \\
\hline $\mathbf{2 0 1 0}$ & $\mathbf{2 0 1 4}$ & $\mathbf{2 0 1 0}$ & $\mathbf{2 0 1 4}$ \\
\hline $51,11 \%$ & $81,48 \%$ & $48,89 \%$ & $18,52 \%$ \\
\hline
\end{tabular}

Seguidamente podemos comprobar la evolución producida en la autopercepción, por parte del profesorado de su nivel de conocimientos y dominio instrumental de las TIC (Tabla 7).

Tabla 7. Evolución de la autopercepción del profesorado sobre su conocimiento sobre TIC

\begin{tabular}{|c|c|c|c|c|c|c|c|}
\hline \multicolumn{2}{|c|}{ BAJO } & \multicolumn{2}{c|}{ USUARIO } & \multicolumn{2}{c|}{ AVANZADO } & \multicolumn{2}{c|}{ EXPERTO } \\
\hline $\mathbf{2 0 1 0}$ & $\mathbf{2 0 1 4}$ & $\mathbf{2 0 1 0}$ & $\mathbf{2 0 1 4}$ & $\mathbf{2 0 1 0}$ & $\mathbf{2 0 1 4}$ & $\mathbf{2 0 1 0}$ & $\mathbf{2 0 1 4}$ \\
\hline $29,55 \%$ & $7,14 \%$ & $54,55 \%$ & $71,43 \%$ & $11,36 \%$ & $21,43 \%$ & $4,55 \%$ & $0 \%$ \\
\hline
\end{tabular}

La evolución en este concepto también es positiva y casi las tres cuartas partes del profesorado que respondió se considera capaz de utilizar las TIC, al menos las 
de uso más cotidiano y común, aunque nuevamente nos surge la duda acerca del conocimiento real sobre otras herramientas y posibilidades que nos ofrecen las TIC.

Uno de los ítems más interesantes para poder calibrar la evolución de las actitudes frente a las TIC es el que se pide a los docentes que señalen el grado de acuerdo con una serie de afirmaciones. Los resultados obtenidos se recogen en la siguiente tabla (Tabla 8):

Tabla 8. Acuerdo con las afirmaciones sobre las TIC

\begin{tabular}{|c|c|c|c|c|c|c|c|c|}
\hline & \multicolumn{2}{|c|}{ Muy de acuerdo } & \multicolumn{2}{|c|}{ De acuerdo } & \multicolumn{2}{|c|}{ En desacuerdo } & \multicolumn{2}{|c|}{$\begin{array}{c}\text { Muy en } \\
\text { desacuerdo }\end{array}$} \\
\hline & 2010 & 2014 & 2010 & 2014 & 2010 & 2014 & 2010 & 2014 \\
\hline $\begin{array}{l}\text { Estoy muy interesado en } \\
\text { las TIC }\end{array}$ & $26,83 \%$ & $38,10 \%$ & $56,10 \%$ & $52,38 \%$ & $17,07 \%$ & $4,76 \%$ & $0 \%$ & $4,76 \%$ \\
\hline $\begin{array}{l}\text { El alumnado muestra } \\
\text { más interés y motivación } \\
\text { si se utilizan las TIC en al } \\
\text { aula }\end{array}$ & $17,07 \%$ & $19,23 \%$ & $58,54 \%$ & $76,92 \%$ & $24,39 \%$ & $0 \%$ & $0 \%$ & $3,85 \%$ \\
\hline $\begin{array}{l}\text { Las TIC facilitan el } \\
\text { aprendizaje autónomo } \\
\text { del alumnado }\end{array}$ & $5 \%$ & $5,56 \%$ & $55 \%$ & $50 \%$ & $37,50 \%$ & $27,78 \%$ & $2,5 \%$ & $16,67 \%$ \\
\hline $\begin{array}{l}\text { Las TIC favorecen un es- } \\
\text { tilo docente más flexible } \\
\text { y personalizado }\end{array}$ & $5 \%$ & $17,65 \%$ & $40 \%$ & $58,82 \%$ & $55 \%$ & $17,65 \%$ & $0 \%$ & $5,88 \%$ \\
\hline $\begin{array}{l}\text { Las TIC permiten un es- } \\
\text { tilo docente más partici- } \\
\text { pativo }\end{array}$ & $2,70 \%$ & $6,25 \%$ & $37,84 \%$ & $68,75 \%$ & $59,46 \%$ & $25 \%$ & $0 \%$ & $0 \%$ \\
\hline $\begin{array}{l}\text { Las TIC fomentan la } \\
\text { capacidad creativa del } \\
\text { alumnado }\end{array}$ & $5,41 \%$ & $10,53 \%$ & $48,75 \%$ & $57,89 \%$ & $43,24 \%$ & $21,05 \%$ & $2,70 \%$ & $10,53 \%$ \\
\hline $\begin{array}{l}\text { Las TIC fomentan el tra- } \\
\text { bajo en grupo y colabo- } \\
\text { rativo }\end{array}$ & $5,71 \%$ & $8,33 \%$ & $34,29 \%$ & $50 \%$ & $57,14 \%$ & $41,67 \%$ & $2,86 \%$ & $0 \%$ \\
\hline $\begin{array}{l}\text { Pienso que las TIC tie- } \\
\text { nen grandes potenciali- } \\
\text { dades educativas }\end{array}$ & $19,05 \%$ & $28 \%$ & $69,05 \%$ & $60 \%$ & $9,52 \%$ & $8 \%$ & $2,38 \%$ & $4 \%$ \\
\hline
\end{tabular}

Como primer comentario a los resultados obtenidos podemos indicar que la actitud del profesorado ante las TIC evoluciona de forma positiva: las afirmaciones que demuestran una actitud más positiva hacia las TIC mejoran en su valoración. Aumenta el interés en las TIC, resaltando el alto porcentaje de respuestas positivas frente a esta afirmación.

En la Tabla 9 se recogen la evolución de las dificultades reconocidas por el profesorado a la hora de la incorporación de las TIC a su tarea docente. 
Tabla 9. Incorporación de las TIC en la práctica docente

\begin{tabular}{|c|c|c|}
\hline & 2010 & 2014 \\
\hline Falta de tiempo para dedicar a las TIC & $84,44 \%$ & $64,29 \%$ \\
\hline Carencia de personal especializado en el centro & $11,11 \%$ & $0 \%$ \\
\hline Escasa motivación del profesorado para el uso de TIC & $26,67 \%$ & $17,86 \%$ \\
\hline Carencia de recursos tecnológicos en el centro & $11,11 \%$ & $3,57 \%$ \\
\hline Desconocimiento para su uso en mi área docente & $31,11 \%$ & $21,43 \%$ \\
\hline Dispersión de la información en Internet & $22,22 \%$ & $10,71 \%$ \\
\hline Falta de recursos educativos disponibles en Internet & $8,89 \%$ & $0 \%$ \\
\hline Bajo nivel de formación en TIC del profesorado & $51,11 \%$ & $39,29 \%$ \\
\hline
\end{tabular}

En general, se ven menos dificultades a la hora de incorporar las TIC en la labor docente, aunque la falta de tiempo y la falta de formación siguen siendo las dificultades que se perciben de manera predominante en el profesorado. La falta de tiempo se señala de manera muy importante por los encuestados (64\%), aunque ha disminuido mucho frente al $84 \%$ de dos años antes.

\section{Conclusiones}

A la hora de establecer conclusiones creemos necesario, por razones de claridad, el diferenciar los aspectos relacionados con las actitudes del profesorado y por otro los del uso de las TIC.

\section{Actitudes del Profesorado}

A partir de los resultados obtenidos en el análisis realizado podemos afirmar que en el centro existe un grupo de profesores altamente motivado hacia las TIC y su utilización en las aulas, con una actitud positiva hacia lo que significan o pueden significar en la sociedad y en la educación. Además ese núcleo es numéricamente muy numeroso.

Otra idea interesante que podemos extraer es que son muy pocas las respuestas con valores bajos (parcialmente en desacuerdo o totalmente en desacuerdo) lo que corrobora lo anteriormente expuesto. Curiosamente, son las variables que nos hablan del tipo de aprendizaje que las TIC pueden propiciar en los alumnos y alumnas (por ejemplo, "Las TIC fomentan la capacidad creativa del alumnado" y "Las TIC fomentan el trabajo en grupo y colaborativo") las que tienen situaciones más dispersas en las respuestas, lo que nos pueden hablar de la necesidad de incidir en dar a conocer esas posibilidades y en cómo se pueden llevar a las aulas.

En lo que se refiere a la valoración de las TIC creemos que es muy destacable el elevado interés hacia las TIC que presenta el profesorado, muy en consonancia con lo que se puede encontrar en otros estudios acerca de la actitud de los docentes frente a las TIC (Barajas et al., 2002; Gargallo et al., 2003; Knezek et al., 2000; Van Braak, 2001; Peralta, 2002; Kollias, 2002).

Son positivas también las actitudes hacia la posibilidad de recibir información y hacia los efectos motivadores de las TIC en los alumnos y alumnas. 
Por lo que podemos observar en el centro, más allá de la dicotomía que hace algunos años se planteaba entre docentes tecnófilos y docentes tecnófobos (García Aretio, 2007), existen docentes "consumidores" de las TIC, que las utilizan de manera dispar en su labor, pero en los que no se percibe especial rechazo, ni siquiera en aquellos que presentan un uso menor en su tarea profesional.

\section{Uso de las TIC}

Las TIC se usan fundamentalmente para el trabajo personal de preparación de las clases pero muy poco en el uso didáctico directo con los alumnos durante las clases. Por eso son la búsqueda de información a través de Internet o el uso del procesador de texto las herramientas que más se usan. Estos resultados coinciden con los de otros estudios realizados y refuerzan la idea de que uno de los mayores esfuerzos que debe hacerse es la formación que posibilite el uso pedagógico de las TIC. Podemos afirmar que se utilizan para la gestión administrativa del aula (ausencias, retrasos...), como elementos que sirven para reforzar las metodologías tradicionales o como una fuente inagotable de información. Aplicaciones todas ellas muy lejos de las enormes posibilidades educativas que nos ofrece la Web 2.0 (De la Torre, 2006).

La proximidad del medio a utilizar es un factor importante a la hora de usarlo, es decir, aquellos elementos que tenemos en el aula son los que se utilizan en mayor medida. Las cuatro paredes del aula sigue siendo el hábitat natural en el que nos encontramos a gusto, eso significa que los medios TIC deben Ilevarse a las aulas cotidianas y no crear nuevos espacios para albergarlos.

Otra idea a destacar que podemos observar es que la conciencia del profesorado respecto a su competencia es bastante buena, ya que se consideran competentes (nivel usuario) en el uso de las TIC, aunque cabe preguntarse si esa percepción no se deberá a que lo que existe es un desconocimiento grande de las posibilidades que nos ofrecen las TIC, de lo que se puede hacer con ellas.

Como refuerzo de lo antes expuesto la gran mayoría de los profesores ve a las TIC como un complemento a los recursos tradicionales, lo que significa que o no son conscientes de las potencialidades educativas de las TIC y la posibilidad de cambio que ofrecen o si lo son las dificultades que perciben son tan grandes que hacen que se mantengan en las líneas pedagógicas tradicionales. Par superar esta situación debe existir una enorme labor de concienciación e información y también proyectos ilusionantes cercanos a la realidad del profesor y que les implique de manera directa.

Como ideas más relevantes de este análisis que hemos realizado, podemos afirmar que existe entre los docentes una "gran clase media", con usos de las TIC bastante asumidos, tanto en lo personal como en lo educativo, con unos poquitos "pioneros" en el camino del uso de las TIC y también con unos poquitos "rezagados" de este proceso.

Esta realidad es importante para este proceso de integración de las TIC en el centro, ya que demuestra una cierta cohesión en el claustro en cuanto al uso de las TIC, que puede evitar que aparezcan tensiones importantes al integrar las TIC en el centro. De esta manera se puede realizar un trabajo más parejo, apoyado en la buena predisposición y actitud positiva demostrada por el profesorado. 


\section{REFERENCIAS BIBLIOGRÁFICAS}

Area, M. (2012). La alfabetización en la Sociedad Digital. En Alfabetización digital y competencias informacionales. Madrid: Fundación Telefónica-Ariel.

Area, M., Gros, B., y Marzal, M.A. (2008). Alfabetizaciones y Tecnologías de la Información y la Comunicación. Madrid: Síntesis.

Calderón, P., y Piñeiro, N. (2007). Actitudes de los docentes ante el uso de las tecnologías educativas. Implicaciones afectivas. Monografías.com.

Carr, W., y Kemmis, S. (1988). Teoría crítica de la enseñanza. Barcelona: Martínez Roca.

Colás, P., y Casanova, J. (2010). Variables docentes y de centro que generan buenas prácticas con TIC. TESI, 11(3), 121-147.

Díez, M. C. (1998) El oficio del maestro es aprender. Cuadernos de Pedagogía, 266, 58-61.

Gros, B. (2000). El ordenador invisible. Hacia la apropiación del ordenador en la enseñanza. Barcelona: Gedisa.

Elliot, J. (1990). La Investigación-Acción en educación. Madrid: Morata.

Ertmer, P. (2005). Teacher Pedagogical Beliefs: The Final Frontier in our Quest for Technology Integration? Educational Technology Research and Development, 53(4), 25-39.

Fidalgo, A. (2011). La innovación docente y los estudiantes. La Cuestión Universitaria, 7.

Hew, K., y Brush, T. (2007). Integrating Technology into K-12 Teaching and Learning: Current Knowledge Gaps and Recommendations for Future Research. Education Technology Research and Development, 55, 223-252.

Inan, F., y Lowther, D. (2010). Laptops in the K-12 Classrooms: Exploring Factors Impacting Instructional Use. Computers \& Education, 58(2), 137-154.

INTEF (2014). Marco Común de Competencia Digital Docente. Borrador con propuestas de descriptores v1.0. Recuperado de http://blog.educalab.es/intef/2014/02/21/ jornada-de-trabajo-sobre-marco-comun-de-competencia-digital-docente/.

Tejedor, F.J., García-Valcárcel, A., y Prada, S. (2009). Medida de actitudes del profesorado universitario hacia la integración de las TIC. Comunicar, 33, 115-124. doi: 10.3916/c33-2009-03-002.

Tirado-Morueta, R., y Aguaded-Gómez, J. I. (2014). Influencias de las creencias del profesorado sobre el uso de la tecnología en el aula. Revista de Educación, 363, 230-255. doi: 10.4438/1988-592X-RE-2012-363-179. 